Original Research Article

\title{
Drug utilization study in post operative patients in surgical ward of tertiary care teaching hospital
}

\author{
Uzma Asiya Samreen ${ }^{1}$, Tejaswi C. H. Reddy ${ }^{1}$, Puchhakayala Goverdhan $^{2 *}$, Mounika Chiluka ${ }^{1}$
}

${ }^{1}$ Student, ${ }^{2}$ Department of Clinical Pharmacy, Vaagdevi

Collage of Pharmacy, Kakatiya

University, Mahatma Gandhi

Memorial Hospital, Warangal,

Telangana, India

Received: 28 January 2019

Accepted: 28 February 2019

\section{*Correspondence to:}

Dr. Puchhakayala Goverdhan,

Email: gov_ku@yahoo.co.in

Copyright: (C) the author(s), publisher and licensee Medip Academy. This is an openaccess article distributed under the terms of the Creative Commons Attribution NonCommercial License, which permits unrestricted noncommercial use, distribution, and reproduction in any medium, provided the original work is properly cited.

\begin{abstract}
Background: This study was aimed to evaluate the drug prescription pattern and to analyze the drug usage in post operative surgical patients with appendicitis, cellulitis, diabetic foot ulcer, hernia and hydrocele in tertiary care teaching hospital.
\end{abstract}

Methods: A prospective observational study was conducted over a period of six months in post-operative surgical patients at Mahatma Gandhi Memorial Hospital, Warangal, Telangana, India, which was approved by Institutional Ethics Committee. The data obtained was analyzed using graph pad prism 7.0 and the conclusions were drawn using descriptive analysis.

Results: A total of 429 cases were recruited during the following study period of which, parenteral drugs were pantoprazole 399 (93\%), ceftriaxone 319 (74.35\%), metronidazole 239 (55.71\%). Oral drugs were multivitamin 400 (93.24\%), serratiopeptidase 299 (69.69\%), voveran $162(37.76 \%)$. Majority of the drugs prescribed were intravenous fluids, antacids, antibiotics, analgesics were accounted. The percentage encounters with generic drugs was $99 \%$ in present study.

Conclusions: The present study provides valuable insight about the overall pattern of drug use profile in postoperative patients in the surgical unit of a tertiary care hospital. The study is useful in decreasing the irrational prescription, which helps to decrease the morbidity and health care burden in the society. The prescribing pattern was according to the National Essential Drug List is very low and needs improvement.

Keywords: Drug utilization, Post-operative patients, Surgical ward

\section{INTRODUCTION}

\section{Drug Utilization Evaluation (DUE)}

Drug Utilization Evaluation ( DUE ), was originally known as Drug Utilization Review (DUR) or Medication Utilization Evaluation (MUE), are an ongoing, authorized and systematic quality improvement process of health care provider prescribing, pharmacist dispensing and patient use of medication. ${ }^{1}$ To ensure proper medical decision making and positive patient outcomes. DUEs involve a complete review of patients medication and prescription data before, during and after dispensing. ${ }^{2}$
DUR is typically classified into three different categories:

- Prospective

- Concurrent

- Retrospective

\section{Prospective DUR}

Prospective DUR is a patient's planned drug therapy before a medication is dispensed. ${ }^{3}$ Imminent audits are routinely performed by Clinical drug specialists in their everyday rehearse by breaking down a physician endorsed prescription measurement and checking on and 
coordinating patient data for conceivable medication collaborations or copy treatment. ${ }^{4}$

\section{Issues commonly addressed by prospective DUR}

- Drug - dosage modification

- Inappropriate duration of drug treatment

- Drug-drug interactions

- Drug - allergy interactions

- Clinical misuse/abuse

- Incorrect drug dosage

- Formulary substitutions (e.g., therapeutic interchange, generic substitution)

\section{Concurrent DUR}

A Concurrent DUR is performed during the course of treatment; it includes continuous monitoring of medication treatment to assist positive patient results. ${ }^{5}$ It is an ongoing process. The clinical pharmacist identifies the dose as inappropriate during their regular treatment chart review and alerts the prescribes about the potential problem. ${ }^{6}$

\section{Issues commonly addressed by concurrent DUR}

- Drug - drug interactions

- Drug - dosage interactions

- Drug - patient precautions (age, gender, pregnancy etc.)

- Therapeutic interchange

- Drug- disease interactions

- Over and under utilization

- Duplicate therapy

\section{Retrospective DUR}

A Retrospective DUR involves reviewing prescribed drugs after the drug is dispensed to the patients. ${ }^{7}$ In this method the medical charts or records of the patients are examined to determine whether the drug therapy met approved criteria and aids prescribers in improving care for their patients either separately or in a specific target population such as patients with diabetes, asthma or hypertension. ${ }^{8}$

The main advantage of this method is the ease of data collection, as records are accessed at the data collector's convenience. ${ }^{5}$

\section{Issues commonly addressed by retrospective DUR}

- Appropriate generic use

- Clinical abuse/misuse

- Drug-disease contraindications

- Drug-drug interactions

- Inappropriate duration of treatment

- Therapeutic appropriateness and/or duplication

- Use of formulate medications whenever appropriate
According to WHO the drug utilization is defined as "the marketing, distribution, prescription and use of drugs in society with special prominence on social, medical and economic consequences". The primary aim of drug utilization research in the population is to facilitate rational drug usage. It is difficult to measure for better changes in the prescription habits due to lack of knowledge. ${ }^{9}$

Clinical practice shows an irrational prescription of drug occurrence. Important reason being lack of knowledge about drugs, unethical drug promotions and irrational prescribing habits by clinicians. The associated problems and feedback are provided to the prescribe Rs. about the monitoring of prescriptions and drug utilization studies. ${ }^{10}$

The majority of the population satisfy the essential drugs in their health care conditions at all the times inadequate situations, exactly which drugs are regarded as essential remains a national responsibility. ${ }^{11}$ Postoperative utilization of drugs is very much marketed.

Also, there are very few studies which describe the utilization of drugs postoperative and their cost. ${ }^{12}$ Drugs are prescribed for the purpose of analgesia, prevention of infection, gastric secretion, nausea, and vomiting, to maintain the hemodynamic status. ${ }^{13}$

The incidence of postoperative nausea and vomiting is estimated to be $25 \%-30 \% .^{14}$ The risk of postoperative nausea and vomiting is multifactorial and can be influenced by patient characteristics, type of surgical procedure, and anesthesia. ${ }^{15}$

Analgesics play an important role in postoperative therapy, in the reduction of post operative complications and hospital stay. ${ }^{16}$

Present study conducted a drug utilization pattern in postoperative patients.

\section{METHODS}

A prospective and concurrent observational study was conducted over a period of 6 months in surgical ward in post operative patients who underwent surgery for Appendicitis, Cellulitis, Diabetic Foot Ulcer, Hernia, Hydrocele at Mahatma Gandhi Memorial Hospital, Warangal, Telangana, India. The institute is a 1000 bed tertiary care hospital equipped with modern diagnostic and treatment facilities. As per selection criteria total 429 patients were enrolled in the study.

\section{Inclusion criteria}

- The patients who underwent major operative procedure on appendicitis, cellulitis, diabetic foot ulcer, hernia and hydrocele were recruited in this study.

- Patients of both genders with age between 18-85 years. 
- All the post operative patients till discharge were taken.

- Drugs used post operatives were only considered and not the drugs that are already going on.

- Patients who are willing to participate and given informed consent form.

\section{Exclusion criteria}

- All Paediatric patients were excluded.

- Pregnant and lactating women were excluded.

- Any patients who dies post operatives before being discharged.

- Patients who absconded/discharged against medical advice.

\section{Protocol preparation and ethical committee approval}

Protocol was prepared according to criteria of the study and submitted, which was reviewed and approved by Institutional Ethical Committee MGM/VCOP/PHARM D/V/014/2017. Informed consent data was obtained from all the patients by explaining them in their language about the process of the work, permission was acquired from Medical Superintendent of Mahatma Gandhi Memorial Hospital (MGMH) Warangal.

\section{Data analysis}

Data of the patients matching inclusion criteria were only recorded. Data like name, age, gender, diagnosis, social history, present medication and post operative medication was recorded from patient case file.

Data was recorded in previously prepared case proforma (which is well suited for DUE) and it is analyzed using MS EXCEL and using GRAPH PAD PRISM (version 7.0) and the conclusions were drawn using descriptive analysis. The results were expressed as proportions or as mean \pm standard deviation and utilization of drugs in surgical ward was evaluated.

\section{RESULTS}

In the present study, total numbers of patients were 429 , out of which 340 (79\%) case files were of male patients and $89(21 \%)$ cases were of female patients, the gender distribution of the sample population of our study was as shown in the Figure 1.

Present data shows the mean age of the patients was 49.51 (18-95 years). 27 patients $(6.29 \%)$ were in the age group of $18-20$ years, 49 patients (11.42\%) were in the age group of 21-30 years, 71 patients $(16.55 \%)$ were in the age group of $31-40$ years, 66 patients $(15.38 \%)$ were in the age group of $41-50$ years, 115 patients $(26.80 \%)$ were in the age group of 51-60years, 74 patients (17.24\%) were in the age group of $61-70$ years, 19 patients $(4.42 \%)$ were in the age group of $71-80$ years, 07 patients $(1.63 \%)$ were in the age group of 81-90 years, 01 patient $(0.23 \%)$ were in the age group of 91-95years.out of 429 cases 158 cases (36\%) were smokers, 278 cases were alcoholics $(64 \%)$ and the mean number of patients during post operative surgical period was $16.67 \pm 4.212$. Of the total cases, 87 patients have undergone surgery for appendicitis, 102 patients for cellulitis, 34 patients for diabetic foot ulcer, 134 patients for hernia, 58 patients for hydrocele, 15 patients who underwent surgery for both hernia and hydrocele was shown in the Table 1.

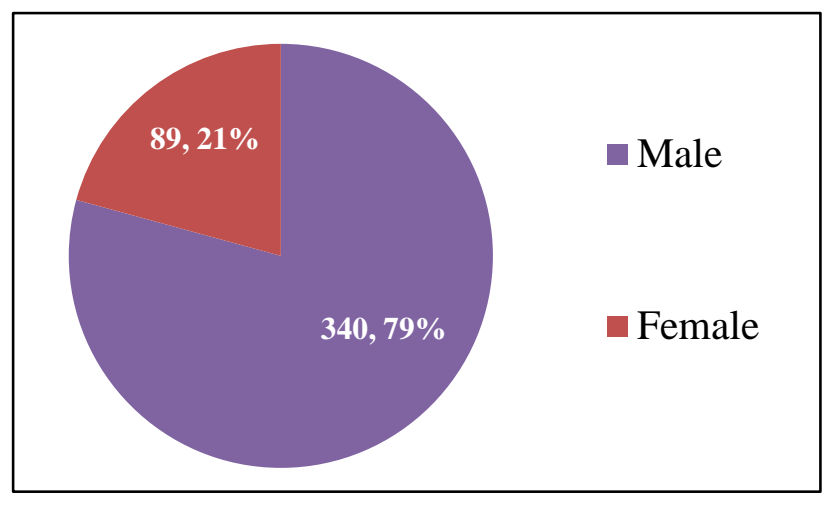

Figure 1: Percentage of gender distribution in drug utilization study.

Table 1: Demographic distribution of age, social habits and disease in drug utilization study.

\begin{tabular}{|lll|}
\hline $\begin{array}{l}\text { Age groups (in } \\
\text { years) }\end{array}$ & $\begin{array}{l}\text { Number of } \\
\text { patients }\end{array}$ & Percentage \\
\hline $11-20$ & 27 & $6.29 \%$ \\
\hline $21-30$ & 49 & $11.42 \%$ \\
\hline $31-40$ & 71 & $16.55 \%$ \\
\hline $41-50$ & 66 & $15.38 \%$ \\
\hline $51-60$ & 115 & $26.80 \%$ \\
\hline $61-70$ & 74 & $17.24 \%$ \\
\hline $71-80$ & 19 & $4.42 \%$ \\
\hline $81-90$ & 07 & $1.63 \%$ \\
\hline $91-100$ & 01 & $0.233 \%$ \\
\hline Social habits & & \\
\hline Smokers & 158 & $64 \%$ \\
\hline Alcoholics & 278 & $36 \%$ \\
\hline & Number of & $\begin{array}{l}\text { Number of } \\
\text { female } \\
\text { patients }\end{array}$ \\
\hline Disease & male patients & 29 \\
\hline Appendicitis & 58 & 29 \\
\hline Cellulitis & 72 & 07 \\
\hline Diabetic foot ulcer & 27 & 24 \\
\hline Hernia & 110 & 00 \\
\hline Hydrocele & 58 & 00 \\
\hline Hernia + Hydrocele & 15 & \\
\hline
\end{tabular}

An injection was prescribed in all the 429 patients either as an injectable antibacterial agent, or an injectable analgesic or intravenous fluid. Thus, the percentage of patients with an injection prescribed was $100 \%$ in our study. Present 
study data shows 17 parenteral medications along with the mean value of parenterals used during post operative surgical period was $127.8 \pm 143.8$ drugs, along with the percentages as shown in the Figure 2.

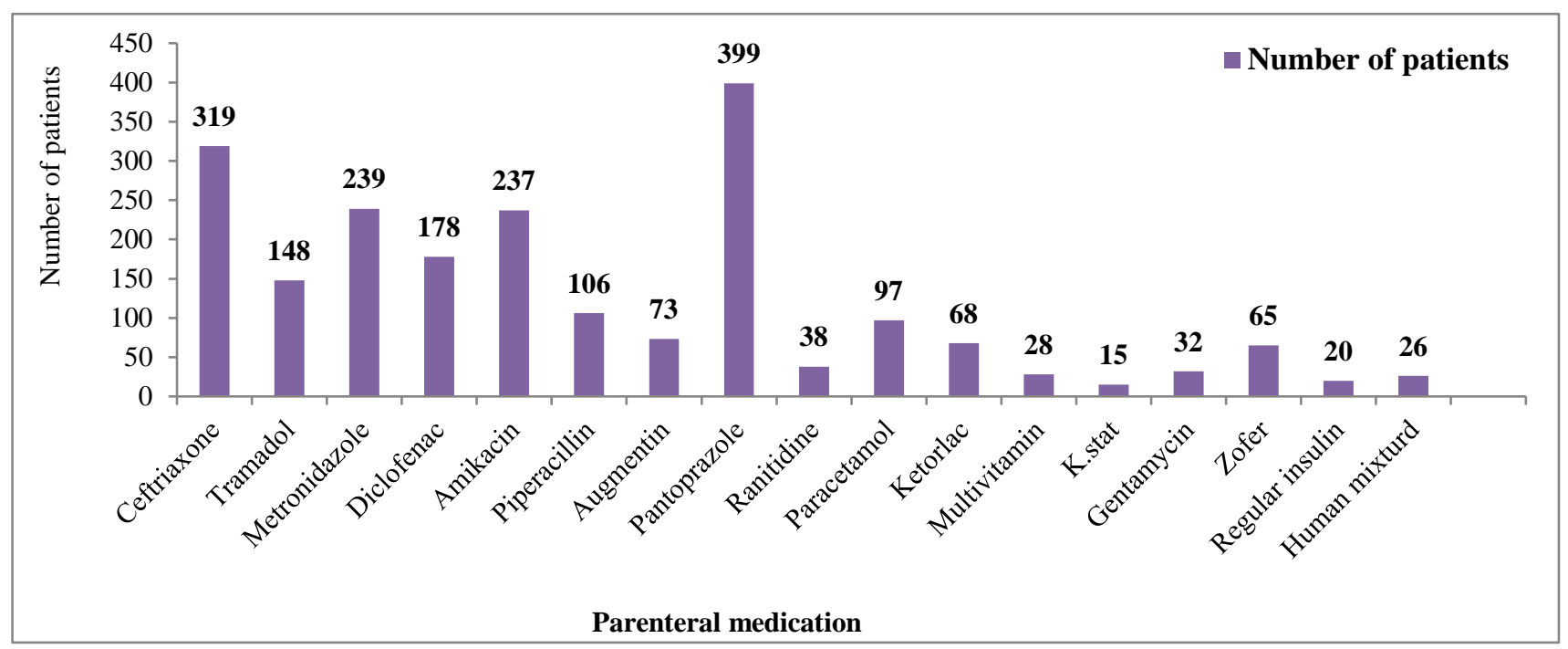

Figure 2: Parenteral medication among patients in drug utilization study.

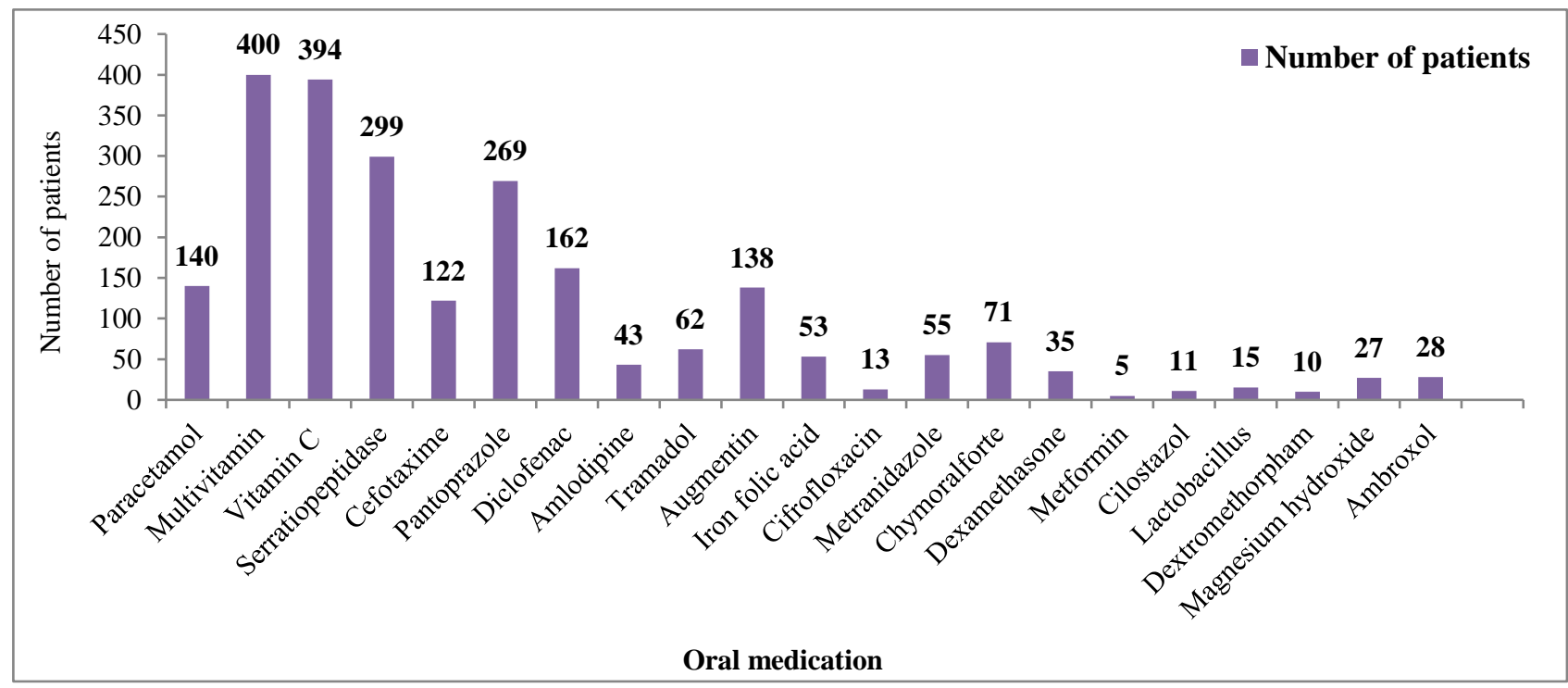

Figure 3: Oral medication among patients in drug utilization study.

Present study data shows 21 oral medications along with mean value of oral drugs used during post operative surgical period was $106.90 \pm 123.87$ drugs, along with the percentages as shown in the Figure 3.

\section{DISCUSSION}

This prospective study attempts to assess general pattern of how drugs were used in surgical ward which was conducted for duration of 6 months. Authors have collected data of 429 patients matching inclusion criteria that underwent surgery and were in their post operative period till they were discharged in the surgery ward of our hospital. The largest proportion was male (79.25\%) which was in accordance with the study done by Bhansali et al, who concluded that $(60 \%)$ of patients were male. ${ }^{12}$ Also, the study done by Ali et al, who concluded that $(58.85 \%)$ of patients were male. ${ }^{17}$

Present study shows the most affected patients were in the age group of 51-60 years (115 patients $(26.80 \%)$ ) which 
was compared to Akhilesh et al, which showed the age group of 20-30 years (95 patients $(29.6 \%)$ ) in their study. ${ }^{18}$

In present study, out of 429 patients the maximum number of patients were diagnosed with hernia 134 (31.23\%) and minimum number i.e., $34(7.92 \%)$ patients had diabetic foot ulcer, moreover $87(20.27 \%)$ patients had appendicitis, $101(23.54 \%)$ patients had cellulitis, $58(13.51 \%)$ patients had hydrocele, a similar study was found which showed out of 160 patients the maximum number $33(20.62 \%)$ patients had hernia and minimum number i.e.,11 (6.87\%) patients had other surgeries like diabetic foot ulcer 27 (10.87\%), cellulitis $27(16.25 \%)$, above study was conducted on antibiotic utilization pattern at the surgery department of tertiary care hospital at Maharastra, India in 2017 Asawari et al. ${ }^{19}$

Among the analgesics, diclofenac sodium was the most commonly used analgesic as parenteral and oral route as post operative pain was one of the major problems after surgery, use of an analgesic has almost become mandatory following surgery, the most commonly used analgesic was diclofenac which is consistent with findings of Jitendra et al. $^{20}$

In present study, the most commonly prescribed antimicrobial agent was ceftriaxone, a third generation cephalosporin which was prescribed in 319 patients (74.35\% of cases) second most common being amoxicillin and clavulanate which are a penicillin type antibiotic used to treat a wide variety of bacterial infections which was prescribed in 138 patients (32.16\% of cases), thirdly piperacillin and tazobactam which was prescribed in 106 patients (24.70\% of cases), which is an extended spectrum penicillin antibiotic, our study was comparable to the study conducted by Prashanth et al, which showed ceftriaxone a third generation cephalosporin which was prescribed in 95 patients (22.94\%), second most common being metronidazole which was prescribed in 49 patients (11.83\%) which covers anaerobic infections, thirdly amikacin sulfate, an aminoglycoside which covers gram negative bacteria was prescribed in 44 patients $(10.62 \%)$. This difference may be due to difference in the availability of drugs in different regions. ${ }^{21}$

In present study all the post operative patients were given injections (100\%) which was comparable to findings of Agarwal et al, $(88.13 \%){ }^{22}$ To prevent injection associated infections, injection use needs to be decreased and injection safety must be achieved. Several interventions were conducted worldwide to decrease injection overuse and/or to achieve safer practice.

In present study pantoprazole usage was (93\%) followed by ceftriaxone usage was $(74.35 \%)$ followed by Metronidazole usage was $(55.71 \%)$. Oral drugs were Multivitamin usage was $(93.24 \%)$ followed by vitamin C usage was $(91.84 \%)$ followed be serratiopeptidase usage was $(69.69 \%)$, which was comparable to the study of Lakshmi et al, shows that tramadol was the most frequently used opioid analgesic by intravenous route followed by diclofenac and pantazocine which was $87 \%$, $9 \%$ and $4 \%$ respectively. ${ }^{23}$

\section{CONCLUSION}

To conclude, although this study had a small size it gave us an overall pattern of drug use profile in post operative patients in the surgical unit of a tertiary care hospital. The number of drugs used in our hospital reflects the common disorders for which surgeries were performed and evaluated. The prescription pattern which was according to the National Essential Drug List was low and needs improvement.

The duration of medication was also not in accordance with the standard guidelines and also remains the main concern of the study. The Standard Operating Procedures (SOPs) must be followed by clinicians practicing in surgical department for effective antimicrobial therapy. The antibiotic resistance should be minimized by promoting infection control practices and rational antibiotic utilization. Present study might be useful in introducing the new concept of drug therapy management in Telangana state, it may also be helpful in identifying the new concept of drug therapy management of patients in different disease conditions in critical care area.

From this study we conclude that a wide spectrum of drugs was utilized from various drug classes and there is a scope for improving rational use of drugs. The prescription of generic drugs need to be promoted. Cost evaluation of medication therapy under same title can also be performed for post-operative patients for avoiding unnecessary costing of medication.

\section{ACKNOWLEDGEMENTS}

Authors would like to thank to all the patients who had participated in this project.

Funding: No funding sources

Conflict of interest: None declared

Ethical approval: The study was approved by the Institutional Ethics Committee (MGM/VCOP/PHARM D/V/014/2017)

\section{REFERENCES}

1. Fauci A, Braunwald E, Kasper D, Hauser S, Longo D. Harrison's principles of internal medicine, 17th edition, published by MC Grow Hill Companies. 3035 section. Impact of delayed admission to Intensive Care Units on mortality of critically ill patients - A cohort study. Critical care. 2011;15-R28.

2. Lucienne TQ Cardoso, Cintia MC Grion, Bonametti AM. Impact of delayed admission to Intensive Care Units on mortality of critically ill patients- A cohort study. Critical care. 2011;15-R28. 
3. Purnima A, Vijeesh TS. Importance of Drug utilization evaluation studies in patient health care. IJPP. 2017;3(10):157-9.

4. Ravikumar KG, Miglani BD, A Textbook of pharmacy practice, 2nd ed, CAREER Publications.

5. Parthasarthi G, Karin NH, Milap CN, A Textbook of pharmacy practice, 2nd ed, Universities Press.

6. Tipnis HP, Amrita B, A Textbook of pharmacy practice, 2nd ed, CAREER Publications.

7. Thomas M, Alexander B, Tony S, Andrel Z. Guidelines for implementing drug utilization review program in hospitals. Russia: Management Sciences for Health; 1997.

8. Rekha MM, Mubeena T. A study on role of Doctor of Pharmacy in Drug Utilization Evaluation Pattern Analysis in inpatient units and reporting its co morbidities in a tertiary hospital. Pharmatutor 2017;5(10): 55-62.

9. Seventh report of WHO Expert committee (including the revised Model List of Essential Drugs): The use of essential drug, WHO TRS 867, World Health Organization; 1997. Available at: www.who.int/iris/bitstream/10665/41938/1/WHO_T RS_867.pdf.

10. Shewade GD, Pradhan CS. Auditing of prescriptions in a government teaching hospital and four retail medical stores in Pondicherry. Indian Journal Pharmacol 1998; 30: 408-410.

11. Ninth report of WHO Expert committee: The use of Essential Drugs, WHO TRS 895, World Health Organization: 2000. Available at: www.who.int.iris/bitstream/10665/42373/1/WHOTRS-895.pdf. Accessed July 2018

12. Bhansali NB, Gosai TR, Dholaria NK, Trivedi HR. Drug utilization study in post-operative patients in surgical ward of a tertiary hospital attached with medical college. Der Pharmacia Letter. 2013;5(1):251-257.

13. Rajeshwari, Nagabushan H. Drug utilization study in post operative patients in obstetrics and gynaecology ward tertiary care hospital. Int J Basic Clin Pharmacol. 2016; 5(2)329-34.

14. Van den Bosch JE, Kalkman CJ, Vergouwe Y, Van Klei WA, Bonsel GJ, Grobbee DE, et al. Assessing the applicability of scoring systems for predicting postoperative nausea and vomiting. Anaesthesia. 2005 Apr; 60(4):323-31.

15. Gan TJ. Postoperative nausea and vomiting-can it be eliminated?. JAMA. 2002 Mar 13;287(10):1233-6.

16. Brennan TJ. Pathophysiology of postoperative pain. Pain. 2011 Mar;152(3 Suppl):S33.

17. Ali SA, Soomro AG, Tahir SM, Memon AS. Prospective basic clinical audit using minimal clinical data set. J Ayub Med Coll Abbottabad. 2010 Mar 1;22(1):58-61.

18. Akhilesh GS, Shuchita V, Ghanshyam GR, Chavda KD. Pattern of drug utilization in surgical outpatient department of a teaching hospital located in Western India. Int J Basic Health 2015;5(9)1291-6.

19. Asawari R, Tirzah C, Shreepa C, Atmaram. Antibiotic utilization pattern at the surgery department of a tertiary care hospital. Asian J Pharmaceut Clin Res. 2017;10(6)131-4.

20. Jitendra MA, Praveen C, Supriya DM, Varsha JP. Drug utilization pattern in acute coronary syndrome at tertiary care hospital: a prospective cross-sectional observational study. Int $\mathrm{J}$ Basic Clin Pharmacol. 2016;5(2)513-6.

21. Prashanth P, Narendranath S, Geetha M, Kumar MN, Patil N, Reshma SR. Trends in prescribing antimicrobials in post-operative wards in a tertiary care hospital. Drug Invention Today. 2011 Dec $1 ; 3(12)$.

22. Agrawal JM, Patel NM, Vaniya HV, Trivedi HR, Balat JD. Drug utilization study in post-operative patients in obstetrics and gynaecology ward of a tertiary care teaching hospital. J Clin Exp Res. 2014;2:103-9.

23. Lakshmi ST, Jaya JS, Mahendra T, Sam JK. Drug utilization evaluation for postoperative patients in obstetrics and gynaecology department in a tertiary care hospital. WJPPS. 2016;5(7):1342-56.

Cite this article as: Samreen UA, Reddy $\mathrm{TCH}$, Goverdhan P, Chiluka M. Drug utilization study in post operative patients in surgical ward of tertiary care teaching hospital. Int J Basic Clin Pharmacol 2019;8:811-6. 